International Journal of Pure and Applied Mathematics

Volume 93 No. 4 2014, 491-499

ISSN: 1311-8080 (printed version); ISSN: 1314-3395 (on-line version)

url: http://www.ijpam.eu

doi: http://dx.doi.org/10.12732/ijpam.v93i4.1

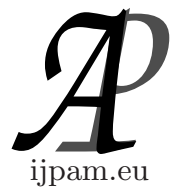

\title{
A NEW AVERAGE METHOD FOR SOLVING
} INTUITIONISTIC FUZZY TRANSPORTATION PROBLEM

\author{
A. Nagoor Gani ${ }^{1}$, S. Abbas ${ }^{2}$ \\ ${ }^{1}$ P.G. and Research Department of Mathematics \\ Jamal Mohamed College (Autonomous) \\ Tiruchirappalli, 620 020, Tamilnadu, INDIA \\ ${ }^{2}$ P.G. and Research Department of Mathematics \\ Khadir Mohideen College \\ Adirampattinam, Tamilnadu, INDIA
}

\begin{abstract}
In this paper a new average method is proposed for finding an optimal solution for an intuitionistic fuzzy transportation problem. The main feature of this method is that it requires very simple arithmetical calculations and avoids large number of iterations. An accuracy function to defuzzify Triangular Intuitionistic Fuzzy Number is also used. Based on this new approach, the optimal solution of Intuitionistic Fuzzy transportation problem is obtained. Finally, an illustrative example is given to verify the developed approach.
\end{abstract}

AMS Subject Classification: 03E72, 03F55, 90B06

Key Words: triangular intuitionistic fuzzy number, accuracy function, intuitionistic fuzzy transportation problem

\section{Introduction}

Transportation problem is a particular class of linear programming. It deals with the transportation of a single product manufactured at different plants (supply origins) to a number of different warehouses (demand destinations).

Received: September 26, 2013

(c) 2014 Academic Publications, Ltd. url: www.acadpubl.eu

${ }^{\S}$ Correspondence author 
The objective of the transportation model is to determine the amount to be shipped from each source to each destination so as to maintain the supply and demand requirements at the lowest transportation cost. The objective is to satisfy the demand at destination from the supply constraint at the minimum transportation cost possible.

Atanassov [1] introduced the concept of Intuitionistic Fuzzy Sets (IFS), which is a Generalization of the concept of fuzzy set [1]. Basic arithmetic operations of TIFNs is defined by Deng-Feng Li in [2] using membership and non-membership values. Basic arithmetic operations of TIFNs such as addition, subtraction and multiplication are defined by S.Mahapatra and T.K.Roy in [4], by considering the six tuple number itself. Most of the authors used the membership and non - membership values of TIFNs for ranking. In this paper we have defined ranking of TIFNs using integral value by considering six tuple TIFNs introduced in [6].

The aim of this paper is to find an optimal solution for an intuitionistic fuzzy transportation problem. Here we have considered TIFN and integral value for ranking. This ranking is applied to solve Intuitionistic Fuzzy Transportation Problem. An accuracy function is developed to defuzzify TIFN.

\section{Preliminaries}

Definition 1. (Fuzzy set) A fuzzy set $\tilde{A}$ is defined by $\tilde{A}=\left\{\left(\mathrm{x}, \mu_{\tilde{A}}(x)\right)\right.$ : $\left.\mathrm{x} \in \mathrm{A}, \mu_{\tilde{A}}(x) \in[0,1]\right\}$.In the pair $\left(x, \mu_{\tilde{A}}(x)\right)$, the first element $x$ belong to the classical set $A$, the second element $\mu_{\tilde{A}}(x)$, belong to the interval $[0,1]$, called Membership function

Definition 2. (Triangular fuzzy number) It is a fuzzy number represented with three points as follows: $\tilde{A}=\left(a_{1}, a_{2}, a_{3}\right)$. This representation in interpreted as membership functions

$$
\mu_{\tilde{A}}(x)=\left\{\begin{array}{lll}
\frac{x-a_{1}}{a_{2}-a_{1}} & \text { for } & a_{1} \leq x \leq a_{2} \\
\frac{a_{3}-x}{a_{3}-a_{2}} & \text { for } & a_{2} \leq x \leq a_{3} \\
0 & & \text { Otherwise }
\end{array}\right.
$$

Definition 3. If $\tilde{A}=\left(a_{1}, a_{2}, a_{3}\right)$ is a Triangular Fuzzy Number, then the defuzzified value or the ordinary (crisp) number of $\tilde{A}, A$ is given below:

$$
A=\frac{a_{1}+4 a_{2}+a_{3}}{6}
$$


Definition 4. (The total integral value of triangular fuzzy number) The total integral value of $\tilde{A}$ is

$$
\begin{aligned}
I_{T}^{\alpha}(\tilde{A}) & =\frac{1}{2} \alpha\left(a_{2}+a_{3}\right)+\frac{1}{2}(1-\alpha)\left(a_{1}+a_{2}\right) \\
& =\frac{1}{2}\left[(1-\alpha) a_{1}+a_{2}+\alpha a_{3}\right] .
\end{aligned}
$$

Definition 5. (Ranking fuzzy numbers with total integral value) Let $\tilde{A}=\left(a_{1}, a_{2}, a_{3}\right)$ and $\tilde{B}=\left(b_{1}, b_{2}, b_{3}\right)$ be two triangular fuzzy numbers.

(i) $\quad I^{\alpha} T(\tilde{A})<\quad I_{T}^{\alpha}(\tilde{B})$ for $\alpha \in[0,1]$, then $\tilde{A}<\tilde{B}$

(ii) $\quad I^{\alpha}{ }_{T}(\tilde{A})>\quad I^{\alpha}{ }_{T}(\tilde{B})$ for $\alpha \in[0,1]$, then $\tilde{A}>\tilde{B}$

(i) $I^{\alpha}{ }_{T}(\tilde{A})=\quad I^{\alpha} T(\tilde{B})$ for $\alpha \in[0,1]$, then $\tilde{A}=\tilde{B}$

\section{Definition 6. An Intutionistic fuzzy set(IFS)}

$\tilde{A}^{I}$ in $X$ is given by a set of ordered triples:

$$
\left.\tilde{A}^{I}=\left\{<x, \mu_{\tilde{A}^{I}}(x)\right), \nu_{\tilde{A}^{I}}(x)>/ x \in X\right\}
$$

where $\mu_{\tilde{A}^{I}}, \nu_{\tilde{A}^{I}}: X \rightarrow[0,1]$ are function such that $0 \leq \mu_{\tilde{A}^{I}}(X)+\nu_{\tilde{A}^{I}}(X) \leq 1$ for all $x \in X$. For each $x$ the number $\mu_{\tilde{A}^{I}}(X)$ and $\nu_{\tilde{A}^{I}}(X)$ represent the degree of membership and degree of non membership of the element $x \in X$ to $A \subset X$, respectively.

Definition 7. (Intutionistic fuzzy number) An intuitionistic fuzzy number $\tilde{A}^{I}$ is:

(i) An intuitionistic fuzzy subset of the real line;

(ii) Normal. i.e., there is any $x_{0} \in R$ such that $\mu_{\tilde{A}^{I}}(X)=1$ ( so that $\left.\nu_{\tilde{A}^{I}}(X)=0\right)$;

(iii) Convex for the membership function $\left.\mu_{\tilde{A}^{I}}(X)\right)$

$$
\mu_{\tilde{A}^{I}}(x)\left(\lambda x_{1}+(1-\lambda) x_{2}\right) \geq \min \left(\mu_{\tilde{A}^{I}}\left(x_{1}\right)\right),\left(\mu_{\tilde{A}^{I}}\left(x_{2}\right)\right), x_{1}, x_{2} \in R, \lambda \in[0,1] ;
$$

(iv) Concave for the non-membership function $\nu_{\tilde{A}^{I}}(X)$ )

$$
\nu_{\tilde{A}^{I}}(x)\left(\lambda x_{1}+(1-\lambda) x_{2}\right) \geq \min \left(\nu_{\tilde{A}^{I}}\left(x_{1}\right)\right),\left(\nu_{\tilde{A}^{I}}\left(x_{2}\right)\right), x_{1}, x_{2} \in R, \lambda \in[0,1] .
$$

Definition 8. An Triangular intutionistic fuzzy number(TIFN) $\mu_{\tilde{A}^{I}}$ is an intuitionistic fuzzy set in $R$ with the following membership function 
$\mu_{\tilde{A}^{I}}(X)$ and non membership function $\nu_{\tilde{A}^{I}}(X)$ :

$$
\begin{gathered}
\mu_{\tilde{A}^{I}}(x)=\left\{\begin{array}{lll}
\frac{x-a_{1}}{a_{2}-a_{1}} & \text { for } & a_{1} \leq x \leq a_{2} \\
\frac{a_{3}-x}{a_{3}-a_{2}} & \text { for } & a_{2} \leq x \leq a_{3} \\
0 & & \text { Otherwise }
\end{array}\right. \\
\nu_{\tilde{A}^{I}}(x)=\left\{\begin{array}{lll}
\frac{a_{2}-x}{a_{2}-a_{1}^{\prime}} & \text { for } & a_{1}^{\prime} \leq x \leq a_{2} \\
\frac{x-a_{2}}{a_{3}^{\prime}-a_{2}} & \text { for } & a_{2} \leq x \leq a_{3}^{\prime} \\
1 & & \text { Otherwise }
\end{array}\right.
\end{gathered}
$$

where $a_{1}^{\prime} \leq a_{1} \leq a_{2} \leq a_{3} \leq a_{3}^{\prime}$ and $\mu_{\tilde{A}^{I}}(X)+\nu_{\tilde{A}^{I}}(X) \leq 1$ or $\mu_{\tilde{A}^{I}}(X)=\nu_{\tilde{A}^{I}}(X)$ for all $x \in R$. This TIFN is denoted by $\mu_{\tilde{A}_{(T I F N)}^{I}}=\left\{\left(a_{1}, a_{2}, a_{3}\right):\left(a_{1}^{\prime}, a_{2}, a_{3}^{\prime}\right)\right\}=$ $\left(a_{1}, a_{2}, a_{3} ; a_{1}^{\prime}, a_{2}, a_{3}^{\prime}\right)$

Definition 9. If $\tilde{A}^{I}=\left(a_{1}, a_{2}, a_{3} ; a_{1}^{\prime}, a_{2}, a_{3}^{\prime}\right)$ is a TIFN, then we define

$$
\tilde{A}^{I}=\frac{\left(a_{1}+2 a_{2}+a_{3}\right)+\left(a_{1}^{\prime}+2 a_{2}+a_{3}^{\prime}\right)}{8}
$$

,an accuracy function of $\tilde{A}^{I}$,to defuzzify the given number.

\section{Operations on Triangular Intuitionistic Fuzzy Number}

Let $\tilde{a}^{I}=\left(a_{1}, a_{2}, a_{3} ; a_{1}^{\prime}, a_{2}, a_{3}^{\prime}\right)$ and $\tilde{b}^{I}=\left(b_{1}, b_{2}, b_{3} ; b_{1}^{\prime}, b_{2}, b_{3}^{\prime}\right)$ two triangular intuitionistic fuzzy number then the arithmetic operations on $\tilde{a}^{I}$ and $\tilde{b}^{I}$ as follows:

Addition: $\tilde{a}^{I} \oplus \tilde{b}^{I}=\left(a_{1}+b_{1}, a_{2}+b_{2}, a_{3}+b_{3} ; a_{1}^{\prime}+b_{1}^{\prime}, a_{2}+b_{2}, a_{3}^{\prime}+b_{3}^{\prime}\right)$.

Subtraction: $\tilde{a}^{I} \ominus \tilde{b}^{I}=\left(a_{1}-b_{3}, a_{2}-b_{2}, a_{3}-b_{1} ; a_{1}^{\prime}-b_{3}^{\prime}, a_{2}-b_{2}, a_{3}^{\prime}-b_{1}^{\prime}\right)$,

Multiplication: $\tilde{a}^{I} \otimes \tilde{b}^{I}=\left(a_{1} b_{1}, a_{2} b_{2}, a_{3} b_{3} ; a_{1}^{\prime} b_{1}^{\prime}, a_{2} b_{2}, a_{3}^{\prime} b_{3}^{\prime}\right)$,

\section{Scalar Multiplication:}

(i) If $k>0$ then $k \tilde{a}^{I}=\left(k a_{1}, k a_{2}, k a_{3} ; k a_{1}^{\prime}, k a_{2}, k a_{3}^{\prime}\right)$

(ii) If $k<0$ then $k \tilde{a}^{I}=\left(k a_{3}, k a_{2}, k a_{1} ; k a_{3}^{\prime}, k a_{2}, k a_{1}^{\prime}\right)$. 


\subsection{Ranking of Triangular Intuitionistic Fuzzy Number}

A Triangular Intuitionistic Fuzzy Number $\tilde{A}^{I}=\{(a, b, c) ;(e, b, f)\}$ is completely defined by its membership and non-membership function as follows

$$
\begin{aligned}
& L_{\mu}(X)=\frac{x-a}{b-a} ; a \leq x \leq b \text { and } R_{\mu}(X)=\frac{c-x}{c-b} ; b \leq x \leq c, \\
& L_{\nu}(X)=\frac{b-x}{b-e} ; e \leq x \leq b \text { and } R_{\nu}(X)=\frac{x-b}{f-b} ; b \leq x \leq f .
\end{aligned}
$$

The inverse functions $L^{-1}$ and $R^{-1}$ can be analytically express as given below:

$$
\begin{aligned}
& L_{\mu}^{-1}(h)=a+(b-a) h ; R_{\mu}^{-1}(h)=c-(c-b) h, \\
& L_{\nu}^{-1}(h)=b-(b-e) h ; R_{\nu}^{-1}(h)=b+(f-b) h .
\end{aligned}
$$

\subsection{Total Integral Value of Triangular Intuitionistic Fuzzy Numbers}

(i) Left integral value of the membership function of $\tilde{A}^{I}$ is given by

$$
I_{L}\left(\tilde{A}^{I}\right)=\int_{0}^{1} L_{\mu}^{-1}(h) \mathrm{d} h=\int_{0}^{1}(b+(b-a) h) \mathrm{d} h=\frac{a+b}{2}
$$

(ii) Left integral value of the non - membership function of $\tilde{A}^{I}$ is given by

$$
I_{L}\left(\tilde{A}^{I}\right)=\int_{0}^{1} L_{\nu}^{-1}(h) \mathrm{d} h=\int_{0}^{1}(b-(b-e) h) \mathrm{d} h=\frac{e+b}{2}
$$

(iii) Right integral value of the membership function of $\tilde{A}^{I}$ is given by

$$
I_{R}\left(\tilde{A}^{I}\right)=\int_{0}^{1} R_{\mu}^{-1}(h) \mathrm{d} h=\int_{0}^{1}(c-(c-b) h) \mathrm{d} h=\frac{b+c}{2}
$$

(iv)Right integral value of the non - membership function of $\tilde{A}^{I}$ is given by

$$
I_{R}\left(\tilde{A}^{I}\right)=\int_{0}^{1} R_{\nu}^{-1}(h) \mathrm{d} h=\int_{0}^{1}(b+(f-b) h) \mathrm{d} h=\frac{b+f}{2}
$$

The total integral value of the membership function of $\tilde{A}^{I}$ is given by

$$
I_{T}^{\alpha}\left(\tilde{A}^{I}\right)=\alpha\left(\frac{b+c}{2}\right)+(1-\alpha)\left(\frac{a+b}{2}\right)=\frac{1}{2}[(1-\alpha) a+b+\alpha c]
$$

The total integral value of the non - membership function of $\tilde{A}^{I}$ is given by

$$
I_{T}^{\beta}\left(\tilde{A}^{I}\right)=\beta\left(\frac{f+b}{2}\right)+(1-\beta)\left(\frac{e+b}{2}\right)=\frac{1}{2}[(1-\beta) e+b+\beta f] .
$$




\subsection{Ranking Procedure}

Let $\tilde{A}^{I}=\left(a_{1}, a_{2}, a_{3} ; a_{1}^{\prime}, a_{2}, a_{3}^{\prime}\right)$ and $\tilde{B}^{I}=\left(b_{1}, b_{2}, b_{3} ; b_{1}^{\prime}, b_{2}, b_{3}^{\prime}\right)$ be two TIFN.

(i)If $I_{T}^{\alpha}\left(\tilde{A}^{I}\right)<I_{T}^{\alpha}\left(\tilde{B}^{I}\right)$ and $I_{T}^{\beta}\left(\tilde{A}^{I}\right)<I_{T}^{\beta}\left(\tilde{B}^{I}\right)$, for $\alpha, \beta \in[0,1]$ then $\tilde{A}^{I}<\tilde{B}^{I}$,

(ii)If $I_{T}^{\alpha}\left(\tilde{A}^{I}\right)>I_{T}^{\alpha}\left(\tilde{B}^{I}\right)$ and $I_{T}^{\beta}\left(\tilde{A}^{I}\right)>I_{T}^{\beta}\left(\tilde{B}^{I}\right)$, for $\alpha, \beta \in[0,1]$ then $\tilde{A}^{I}>\tilde{B}^{I}$,

(iii)If $I_{T}^{\alpha}\left(\tilde{A}^{I}\right)=I_{T}^{\alpha}\left(\tilde{B}^{I}\right)$ and $I_{T}^{\beta}\left(\tilde{A}^{I}\right)=I_{T}^{\beta}\left(\tilde{B}^{I}\right)$,for $\alpha, \beta \in[0,1]$ then $\tilde{A}^{I}=\tilde{B}^{I}$.

\section{Solution Algorithm for New Average Method}

Step 1: Construct the transportation model (Table) from the given transportation problem.

Step 2: Subtract each row entries of the transportation table from the respective row minimum and then subtract each column entries of the transportation table from the respective column minimum, so that each row and column will have least one zero.

Step 3: Now there will be at least one zero in each row and column in the reduced cost matrix. Select the first zero (row wise) occurring in the cost matrix. Count the total number of zeros excluding the selected one in the corresponding row and column. Then find the average of corresponding demand and supply for this zero. Repeat the procedure for all zeros in the matrix.

Step 4: Now choose a zero for which the minimum demand supply average and allocate the maximum possible to that cell.

Step 5: After performing step 4 delete the row or column (where supply or demand becomes zero) for further calculation.

Step 6: Check whether the resultant matrix possesses at least one zero in each column and in each row. If not repeat step2, otherwise go to step 7 .

Step 7: Repeat step 3 to step 6 until and unless all the demands are satisfied and all the supplies are exhausted.

Step 8: For the allocated values calculate the optimal cost.

\section{Transportation Problem in Fuzzy Environment}

Step 1: Mathematical Formulation: 


\begin{tabular}{|c|c|c|c|c|c|}
\hline & $D_{1}$ & $D_{2}$ & $D_{3}$ & $D_{4}$ & Supply \\
\hline$S_{1}$ & 13 & 20 & 25 & 8 & $(3,8,10)$ \\
\hline$S_{2}$ & 50 & 20 & 25 & 45 & $(6,10,13)$ \\
\hline$S_{3}$ & 25 & 6 & 55 & 10 & $(8,11,15)$ \\
\hline Demend & $(2,4,7)$ & $(5,7,10)$ & $(3,6,8)$ & $(7,12,13)$ & \\
\hline
\end{tabular}

When applying the above algorithm and ranking procedure, we get the optimum solution for fuzzy transportation problem.

\begin{tabular}{|c|c|c|c|c|c|}
\hline & $D_{1}$ & $D_{2}$ & $D_{3}$ & $D_{4}$ & Supply \\
\hline$S_{1}$ & 13 & 20 & 25 & $\begin{array}{c}8 \\
(-4,4,8)\end{array}$ & $(3,8,10)$ \\
\hline$S_{2}$ & 50 & $\begin{array}{c}20 \\
(-2,4,10)\end{array}$ & $\begin{array}{c}25 \\
(3,6,8)\end{array}$ & 45 & $(6,10,13)$ \\
\hline$S_{3}$ & 25 & $\begin{array}{c}6 \\
(-5,3,12)\end{array}$ & 55 & $\begin{array}{c}10 \\
(-1,8,17)\end{array}$ & $(8,11,15)$ \\
\hline Demend & $(2,4,7)$ & $(5,7,10)$ & $(3,6,8)$ & $(7,12,13)$ & \\
\hline
\end{tabular}

The fuzzy optimal solution for the given fuzzy transportation problem is $(-11,412,797)$, and the crisp value is 405.67 .

\section{Intuitionistic Fuzzy Transportation Problem (IFTP)}

Consider a transportation with m Intuitionistic Fuzzy (IF) origins and $\mathrm{n}$ IF destination. Let $C_{i j}(i=1,2, \cdots, m, j=1,2, \cdots, n)$ be the cost of transporting one unit of the product form ith origin to jth destination .Let $\tilde{a}_{i}^{I}(i=$ $1,2, \cdots, m)$ be the quantity of commodity available at IF origin $i$. Let $\tilde{b}_{j}^{I}$ $(j=1,2, \cdots, n)$ be the quantity of commodity needed of IF destination $\mathrm{j}$. Let $X_{i j}(i=1,2, \cdots, m, j=1,2, \cdots, n)$ is quantity transported from $i^{t h}$ IF origin to $j^{\text {th }}$ destination.

Mathematical Model of Intuitionistic Fuzzy Transportation Problem is

$$
\begin{gathered}
\operatorname{mini} \tilde{Z}_{j}^{I}=\sum_{i=1}^{m} \sum_{j=1}^{n} \tilde{x}_{i j}^{I} \tilde{c}_{i j}^{I}, \\
\sum_{i=1}^{n} \tilde{x}_{i j}^{I}=\tilde{a}_{i}^{I}, \\
\sum_{i=1}^{n} \tilde{x}_{i j}^{I}=\tilde{b}_{j}^{I},
\end{gathered}
$$

$\tilde{x}_{j}^{I} \geq 0$ for all $i$ and $j$. 
The above IFTP can be stated in the below tabular form.

\begin{tabular}{|c|c|c|c|c|c|}
\hline & $D_{1}$ & $D_{2}$ & $\cdots$ & $D_{n}$ & IF Capacity \\
\hline$O_{1}$ & $C_{11}$ & $C_{12}$ & $\cdots$ & $C_{1 n}$ & $\tilde{a}_{1}^{I}$ \\
& $X_{11}$ & $X_{12}$ & $\cdots$ & $X_{1 n}$ & \\
\hline$O_{2}$ & $C_{21}$ & $C_{22}$ & $\cdots$ & $C_{2 n}$ & $\tilde{a}_{2}^{I}$ \\
& $X_{21}$ & $X_{22}$ & $\cdots$ & $X_{2 n}$ & \\
\hline$O_{3}$ & $\vdots$ & $\vdots$ & $\vdots$ & $\vdots$ & $\vdots$ \\
\hline$O_{m}$ & $C_{m 1}$ & $C_{m 2}$ & $\cdots$ & $C_{m n}$ & $\tilde{a}_{m}^{I}$ \\
& $X_{m 1}$ & $X_{m 2}$ & $\cdots$ & $X_{m n}$ & \\
\hline IF Demend & $\tilde{b}_{1}^{I}$ & $\tilde{b}_{2}^{I}$ & & $\tilde{b}_{m}^{I}$ & $\sum \tilde{a}_{i}^{I}=\sum \tilde{b}_{j}^{I}$ \\
\hline
\end{tabular}

Numerical Illustration

\begin{tabular}{|c|c|c|c|c|c|}
\hline & $D_{1}$ & $D_{2}$ & $D_{3}$ & $D_{4}$ & Supply \\
\hline$S_{1}$ & 13 & 20 & 25 & 8 & $(3,8,10 ; 2,8,13)$ \\
\hline$S_{2}$ & 50 & 20 & 25 & 45 & $(6,10,13 ; 4,10,15)$ \\
\hline$S_{3}$ & 25 & 6 & 55 & 10 & $(8,11,15 ; 6,11,18)$ \\
\hline Demend & $(2,4,7 ; 1,4,9)$ & $(5,7,10 ; 3,7,12)$ & $(3,6,8 ; 2,6,10)$ & $(7,12,13 ; 6,12,15)$ & \\
\hline
\end{tabular}

When applying the above algorithm and ranking procedure, we get the optimum solution for intuitionistic fuzzy transportation problem.

\begin{tabular}{|c|c|c|c|c|c|}
\hline & $D_{1}$ & $D_{2}$ & $D_{3}$ & $D_{4}$ & Supply \\
\hline$S_{1}$ & 13 & 20 & 25 & 8 & $(3,8,10 ; 2,8,13)$ \\
& $(2,4,7 ; 1,4,9)$ & & & $(-4,4,8 ;-7,4,12)$ & \\
\hline$S_{2}$ & 50 & $(-2,4,10 ;-6,4,13)$ & $(3,6,8 ; 2,6,10)$ & 45 & $(6,10,13 ; 4,10,15)$ \\
& & 6 & 55 & & $(-1,8,17 ;-6,8,22)$ \\
\hline$S_{3}$ & 25 & $(-5,3,12 ;-10,3,18)$ & & $(8,11,15 ; 6,11,18)$ \\
& & $(5,7,10 ; 3,7,12)$ & $(3,6,8 ; 2,6,10)$ & $(7,12,13 ; 6,12,15)$ & \\
\hline Demend & $(2,4,7 ; 1,4,9)$ & &
\end{tabular}

Step 8: The optimal solution for the given intuitionistic fuzzy transportation problem is $(-11,412,797 ;-233,412,1051)$, and the crisp value is 406.5 .

\section{Conclusions}

The new average method provides an optimal solution in less number of iterations, directly for a given intuitionistic fuzzy transportation problem. Also this method required less number of time and is very easy to understand and apply. So it will be very helpful for decision makers who are dealing with logistic and supply chain problem.

\section{References}

[1] Atanassov.K.T., Intuitionistic fuzzy sets, Fuzzy Sets and Systems, 1986, 87-96.

[2] Deng-Feng-Li, A note on "Using intuitionistic fuzzy sets for fault - tree analysis on printed circuit board assembly", Micro Electronics Reliability, 2008, 48, 1741. 
[3] Ezhil Vannan.S, Rekha.S, A New Method for Obtaining an Optimal Solution for Transportation Problems, IJEAT, Vol- 2, Issue - 2, June 2013, 369-371.

[4] Mahapatra.G.S., and Roy.T.K., Reliability Evaluation using Triangular Intuitionistic Fuzzy numbers Arithmetic operations, World Academy of science, Engineering and Technology 2009, 50, 574-581.

[5] Nagoor Gani.A and Ponnalagu.K, Solving Linear Programming Problem in an Intuitionistic Environment, Proceedings of the Heber international conference on Applications of Mathematics and Statistics, HICAMS 5-7 Jan 2012.

[6] Nagoor Gani.A and Ponnalagu.K, A New Approach on Solving Intuitionistic Fuzzy Linear Programming Problem, Applied Mathematical Sciences, Vol. 6, 2012, no. 70, 3467 - 3474

[7] Nagoor Gani.A and Abbas.S, Solving Intuitionistic Fuzzy Transportation Problem Using Zero Suffix Algorithm, IJMSEA, Vol. 6 No. III, May- 2012, 73-82.

[8] Sudhakar.V.J., Arunsankar.N and Karpagam.T, A New approach for finding an Optimal solution for transportation Problems. European journal of scientific Research, vol 68, pp.254-257, 2012.

[9] Xinfan Wang, Fuzzy number intuitionistic fuzzy arithmetic aggregation operators, International Journal of Fuzzy Systems, 2008, vol.10, (2), 104111. 
